SECTION 2. Applied mathematics. Mathematical modeling.

Naumov Anatoly Aleksandrovich, Docent, candidate of Technical Sciences,

Center of Applied Mathematical Research, Novosibirsk, Russia, e-mail: A_A_Naumov@mail.ru

\title{
TO $s_{p}$-INSTABILITY OF NORMALIZATION OF MULTICRITERIAL CRITERIA METHOD FOR DECIDING OF OPTIMIZATION PROBLEMS
}

In the paper the stability of the method of normalization criteria used to solve multi-criteria optimization problems is studied.

Key words: Multi-criteria problems, method of normalization criteria, sustainability

\section{К $\boldsymbol{S}_{\boldsymbol{p}}$-НЕУСТОЙЧИВОСТИ МЕТОДА НОРМАЛИЗАЦИИ КРИТЕРИЕВ РЕШЕНИЯ МНОГОКРИТЕРИАЛЬНЫХ ЗАДАЧ ОПТИМИЗАЦИИ}

В работе исследован на устойчивость метод нормализачии критериев, используемый для решения многокритериальных задач оптимизации.

Ключевые слова: Многокритериальные задачи, метод нормализачии критериев, устойчивость.

В работе исследован на устойчивость метод нормализации критериев, используемый для решения многокритериальных задач оптимизации (см. [3]-[6]).

Постановка задачи. Предположим, что необходимо решить многокритериальную задачу линейного программирования:

$$
\begin{aligned}
& \mathrm{f}_{1}\left(\mathrm{x}_{1}, \mathrm{x}_{2}, \mathrm{x}_{3}, \ldots, \mathrm{x}_{\mathrm{n}}\right) \rightarrow \max , \\
& \mathrm{f}_{2}\left(\mathrm{x}_{1}, \mathrm{x}_{2}, \mathrm{x}_{3}, \ldots, \mathrm{x}_{\mathrm{n}}\right) \rightarrow \max , \\
& \ldots \\
& \mathrm{f}_{\mathrm{p}}\left(\mathrm{x}_{1}, \mathrm{x}_{2}, \mathrm{x}_{3}, \ldots, \mathrm{x}_{\mathrm{n}}\right) \rightarrow \max ,
\end{aligned}
$$

при ограничениях:

$$
\left\{\begin{array}{c}
\mathrm{g}_{1}\left(\mathrm{x}_{1}, \mathrm{x}_{2}, \mathrm{x}_{3}, \ldots, \mathrm{x}_{\mathrm{n}}\right) \leq \mathrm{b}_{1}, \\
\mathrm{~g}_{2}\left(\mathrm{x}_{1}, \mathrm{x}_{2}, \mathrm{x}_{3}, \ldots, \mathrm{x}_{\mathrm{n}}\right) \leq \mathrm{b}_{2}, \\
\ldots \\
\mathrm{g}_{\mathrm{m}}\left(\mathrm{x}_{1}, \mathrm{x}_{2}, \mathrm{x}_{3}, \ldots, \mathrm{x}_{\mathrm{n}}\right) \leq \mathrm{b}_{\mathrm{m}} .
\end{array}\right.
$$

Предположим, что область допустимых решений задачи (обозначим ее через D), образованная ограничениями (2), является непустой и 
ограниченной. Таким образом, на этой области существуют оптимальные решения задачи для каждого из критериев множества (1).

Основная идея метода нормализации критериев состоит в переходе от многокритериальной задачи (1)-(2) к скалярной задаче следующего вида (см. [3]):

$$
\lambda \rightarrow \max ,
$$

при ограничениях:

где

$$
\left\{\begin{array}{c}
\lambda-\lambda_{1}\left(x_{1}, x_{2}, x_{3}, \ldots, x_{n}\right) \leq 0 \\
\lambda-\lambda_{2}\left(x_{1}, x_{2}, x_{3}, \ldots, x_{n}\right) \leq 0 \\
\ldots \\
\lambda-\lambda_{p}\left(x_{1}, x_{2}, x_{3}, \ldots, x_{n}\right) \leq 0 \\
g_{1}\left(x_{1}, x_{2}, x_{3}, \ldots, x_{n}\right) \leq b_{1} \\
g_{2}\left(x_{1}, x_{2}, x_{3}, \ldots, x_{n}\right) \leq b_{2} \\
\ldots \\
g_{m}\left(x_{1}, x_{2}, x_{3}, \ldots, x_{n}\right) \leq b_{m}
\end{array}\right.
$$

$\lambda=\min \left(\lambda_{1}\left(\mathrm{x}_{1}, \mathrm{x}_{2}, \mathrm{x}_{3}, \ldots, \mathrm{x}_{\mathrm{n}}\right), \lambda_{2}\left(\mathrm{x}_{1}, \mathrm{x}_{2}, \mathrm{x}_{3}, \ldots, \mathrm{x}_{\mathrm{n}}\right), \ldots, \lambda_{\mathrm{p}}\left(\mathrm{x}_{1}, \mathrm{x}_{2}, \mathrm{x}_{3}, \ldots, \mathrm{x}_{\mathrm{n}}\right)\right)$; $\mathrm{f}_{\mathrm{i}}^{\min }, \mathrm{f}_{\mathrm{i}}^{\max }, \mathrm{i}=1,2,3, \ldots, \mathrm{p}$, - это наименьшие и наибольшие значения соответствующих критериев из множества (1) на области допустимых решений (2). Таким образом, нормализация критериев - это прием, сводящий задачу векторной оптимизации к задаче с одним критерием.

Введем основные обозначения для элементов задачи многокритериальной оптимизации. Целевые функции задачи (1) сведем в общий (единый) вектор

$$
\mathrm{F}=\left(\mathrm{f}_{1}\left(\mathrm{x}_{1}, \mathrm{x}_{2}, \mathrm{x}_{3}, \ldots, \mathrm{x}_{\mathrm{n}}\right), \mathrm{f}_{2}\left(\mathrm{x}_{1}, \mathrm{x}_{2}, \mathrm{x}_{3}, \ldots, \mathrm{x}_{\mathrm{n}}\right), \ldots, \mathrm{f}_{\mathrm{p}}\left(\mathrm{x}_{1}, \mathrm{x}_{2}, \mathrm{x}_{3}, \ldots, \mathrm{x}_{\mathrm{n}}\right)\right)
$$

и будем считать, как и выше, что все функции этого вектора необходимо максимизировать. Ограничения (2) объединим во множество $\mathrm{S}=$ $\left\{\mathrm{g}_{1}\left(\mathrm{x}_{1}, \mathrm{x}_{2}, \mathrm{x}_{3}, \ldots, \mathrm{x}_{\mathrm{n}}\right) \leq \mathrm{b}_{1}, \mathrm{~g}_{2}\left(\mathrm{x}_{1}, \mathrm{x}_{2}, \mathrm{x}_{3}, \ldots, \mathrm{x}_{\mathrm{n}}\right) \mathrm{b}_{2}, \ldots, \mathrm{g}_{\mathrm{m}}\left(\mathrm{x}_{1}, \mathrm{x}_{2}, \mathrm{x}_{3}, \ldots, \mathrm{x}_{\mathrm{n}}\right) \leq\right.$ $\left.\mathrm{b}_{\mathrm{m}}\right\}$. Тогда, саму задачу многокритериальной оптимизации обозначим в виде кортежа: $\mathrm{P}=\langle\mathrm{F}, \mathrm{S}\rangle$. Последняя запись читается таким образом: требуется найти максимум векторной функции $\mathrm{F}$ при ограничениях $\mathrm{S}$. Или найти $\mathrm{F} \rightarrow \max$ при ограничениях S. Введем в рассмотрение следующие определения. 
Определение 1. Парето-множество задачи $\mathrm{P}=\langle\mathrm{F}, \mathrm{S}\rangle$ обозначим через $\mathrm{D}_{\pi}$, а множество ограничений задачи его определяющее - через $\mathrm{S}_{\pi}$ и назовем множеством активных ограничений.

Определение 2. Для фиксированного множества ограничений $\mathrm{S}$ (и, следовательно, области D) введем в рассмотрение отображения: $\mathrm{S} \stackrel{\mathrm{F}}{\rightarrow} \mathrm{S}_{\pi} \quad$ и, аналогично, $\mathrm{D} \stackrel{\mathrm{F}}{\rightarrow} \mathrm{D}_{\pi}$.

Определение 3. Множество ограничений из $\mathrm{S}$ не входящее во множество активных ограничений $S_{\pi}$ назовем множеством пассивных ограничений задачи $\mathrm{P}=\langle\mathrm{F}, \mathrm{S}\rangle$ и обозначим через $\mathrm{S}_{\mathrm{p}}$. Таким образом, $\mathrm{S}=$ $S_{\pi} \cup S_{p}$, a $S_{\pi} \cap S_{p}=\varnothing$.

Определение 4. Две задачи $\mathrm{P}_{1}$ и $\mathrm{P}_{2}$ назовем $\boldsymbol{D}_{\boldsymbol{\pi}}$-эквивалентными, если они имеют одинаковые множества Парето, т.е. выполняется $\mathrm{D}_{\pi, \mathrm{P}_{1}}=$ $\mathrm{D}_{\pi, \mathrm{P}_{2}}$. Эквивалентность задач обозначим следующим образом: $\mathrm{P}_{1} \sim_{\mathrm{D}_{\pi}} \mathrm{P}_{2}$.

Заметим, что $\mathrm{D}_{\pi}$-эквивалентные задачи в общем случае могут иметь не совпадающие между собой векторы критериев $\left(\mathrm{F}_{\mathrm{P}_{1}} \neq \mathrm{F}_{\mathrm{P}_{2}}\right)$ и множества ограничений $\left(\mathrm{S}_{\mathrm{P}_{1}} \neq \mathrm{S}_{\mathrm{P}_{2}}\right)$. Аналогично определению для $\mathrm{D}_{\pi}$-эквивалентных задач, можно ввести определения для $\left\langle\mathrm{D}_{\pi}, \mathrm{F}\right\rangle-,\left\langle\mathrm{D}_{\pi}, \mathrm{S}\right\rangle-,\left\langle\mathrm{S}_{\pi}, \mathrm{F}\right\rangle$ эквивалентных задач.

Определение 5. Метод (M) решения задачи $\langle\mathrm{F}, \mathrm{S}\rangle$ - это отображение пары $\langle\mathrm{F}, \mathrm{S}\rangle$ в некоторую область (точку) $\mathrm{x}^{*}$ области $\mathrm{D}_{\pi}$, т.е. $\langle\mathrm{F}, \mathrm{S}\rangle \stackrel{\mathrm{M}}{\rightarrow} \mathrm{x}^{*} \in \mathrm{D}_{\pi}$ или $\mathrm{M}:\langle\mathrm{F}, \mathrm{S}\rangle \rightarrow \mathrm{x}^{*} \in \mathrm{D}_{\pi}$ (для точки $\left.x^{*}\right)$.

Определение 6. Метод (М) решения задачи $(\mathrm{P}=\langle\mathrm{F}, \mathrm{S}\rangle)$ является $\boldsymbol{S}_{\boldsymbol{p}}$ устойчивым, если решение задачи, найденное этим методом, не меняется при изменении элементов множества $S_{p}$. При этом элементы вектора $F$ и множества $\mathrm{D}_{\pi}$ являются фиксированными и не изменяются при изменении $\mathrm{S}_{\mathrm{p}}$. Формально это свойство метода М можно записать таким образом:

$$
\begin{aligned}
& \forall_{\mathrm{S}_{\mathrm{p}_{1}} \neq \mathrm{S}_{\mathrm{p}_{2}}}\left(\mathrm{M}:\left\langle\mathrm{F}, \mathrm{S}=\mathrm{S}_{\pi} \cup \mathrm{S}_{\mathrm{p}_{1}}\right\rangle \rightarrow \mathrm{x}_{1}^{*} \in \mathrm{D}_{\pi} \& \mathrm{M}:\left\langle\mathrm{F}, \mathrm{S}=\mathrm{S}_{\pi} \cup \mathrm{S}_{\mathrm{p}_{2}}\right\rangle \rightarrow \mathrm{x}_{2}^{*} \in\right. \\
\left.\mathrm{D}_{\pi}\right) \Rightarrow & \mathrm{x}_{1}^{*}=\mathrm{x}_{2}^{*} .
\end{aligned}
$$

Отметим, что большинство методов, предназначенных для решения многокритериальных задач оптимизации (см., например, [1], [2]), являются $\mathrm{S}_{\mathrm{p}}$-устойчивыми. Это свойство методов представляется очевидным, поскольку было бы нелогично, если бы решения таких задач зависели от множеств их пассивных ограничений.

Утверждение. Метод нормализации критериев (см. [3]-[6]) не является $\mathrm{S}_{\mathrm{p}}$-устойчивым.

\section{Литература}

1. Кини Р.Л., Райфа Х. Принятие решений при многих критериях: предпочтения и замещения. - М: Радио и связь, 1981. -560 с. 
2. Подиновский В.В., Ногин В.Д. Парето-оптимальные решения многокритериальных задач. - М.: Наука, 1982. - 255 с.

3. Машунин Ю.К. Методы и модели векторной оптимизации. - М.: Наука, 1986. $-140 \mathrm{c}$.

4. Кириллов Ю.В., Назимко Е.Н. Многокритериальная модель оптимизации структуры капитала// Экономический анализ: теория и практика, 2011, № 32 (239), С. 57-63.

5. Кириллов Ю.В., Назимко Е.Н. Многокритериальная задача оптимизации структуры капитала и еe решение в системе Maple// Экономика и менеджмент систем управления, 2013, т. 8, № 2.1, С. 149-160.

6. Кириллов Ю.В., Досужева Е.Е. Многокритериальная экономикоматематическая модель оценки коммерческой эффективности инвестирования// Финансовая аналитика: Проблемы и решения, 2013, № 32, С. $18-24$.

7. Список трудов [Электронный ресурс]. URL: https://sites.google.com/site/ anatolynaumov2011/home/spisok-trudov-list-of-papers (дата обращения: 25.09.2013). 